\title{
Lighting Up Heart Cells with Tiny Lasers
}

\section{A new microscopy technique that uses micrometer-sized lasers can track beating-induced changes in a heart cell's refractive index.}

\section{By Katherine Wright}

N o easy method exists for imaging single cells inside live animals. Marcel Schubert of the University of St Andrews, UK, hopes his newly demonstrated technique could change that. In a talk at the Optical Society's Biophotonics Conference last month, Schubert discussed how he and his colleagues have tracked the beating of heart cells using micrometer- and nanometer-sized lasers placed directly inside the cells. The lasers could allow researchers to monitor

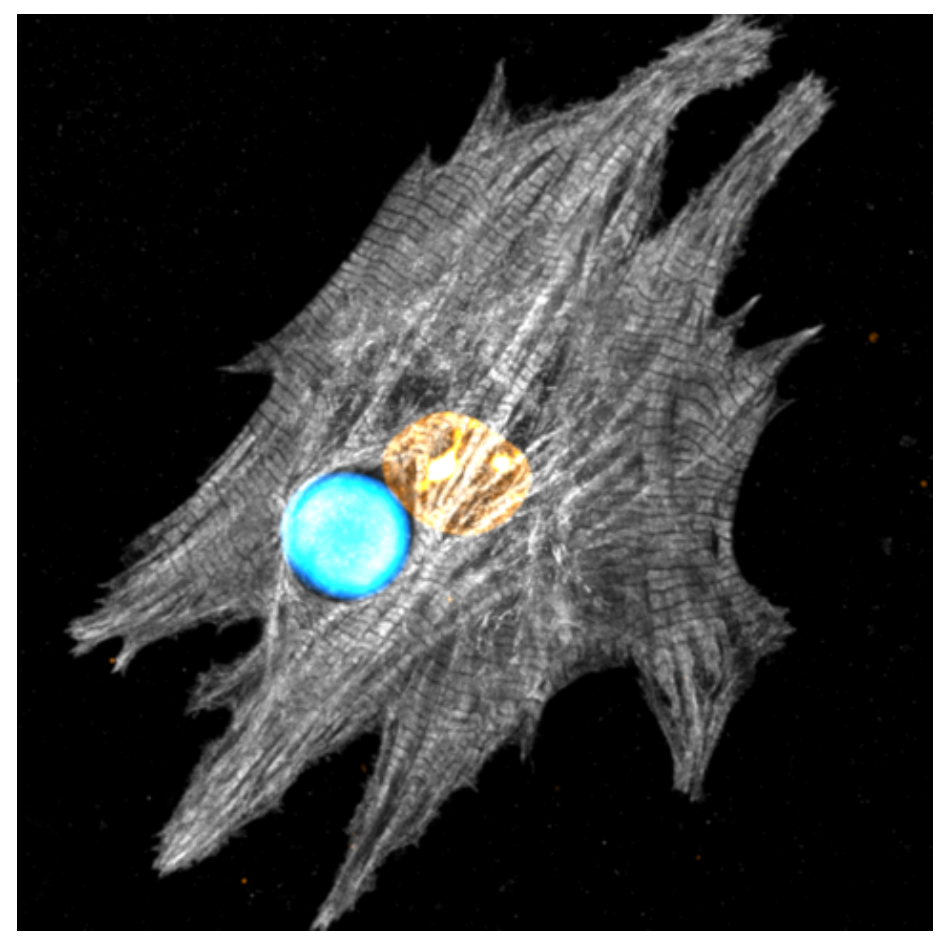

Micrometer-sized lasers placed inside heart cells can track the beating of these dynamics systems.

Credit: M. Schubert/University of St Andrews the dynamics of cells deep inside live tissues.

Medical imaging, such as ultrasound and MRI, can let doctors see inside the body, but the resolution of these in vivo methods is typically too low to make out individual cells. The only real option for viewing cells in living organisms is to use fluorescence-based techniques, Schubert said. In these techniques, a camera records the light emitted from a fluorescent dye in the cells, allowing features of individual cells to be distinguished. Researchers can use fluorescence microscopy to image in vivo brain cells, for example. But the tool is challenging to apply and requires cutting out a piece of the skull and replacing that bone with a porthole-like window to gain direct visual access to the tissue.

As well as the invasive nature of the tool for in vivo applications, fluorescence microscopy has the problem that it can typically only image the outer few cell layers of a tissue. In the heart, for example, light penetrates only about $100 \mu \mathrm{m}$ of tissue. "We really need a brighter light source," Schubert said, which is what he and his colleagues have found.

In their experiments, Schubert and his colleagues don't place dye molecules directly in the cell but rather use a micrometer-sized plastic bead that is infused with dye molecules. This bead is known to act as a laser. The lasing works as follows: A bead is illuminated with light, causing its dye molecules to fluoresce. Because of the bead's specific refractive properties, most of this fluorescent light gets trapped inside the bead, circling around the bead's outer surface.

For certain wavelengths, this circling light constructively interferes, building in intensity. The intensity also increases 

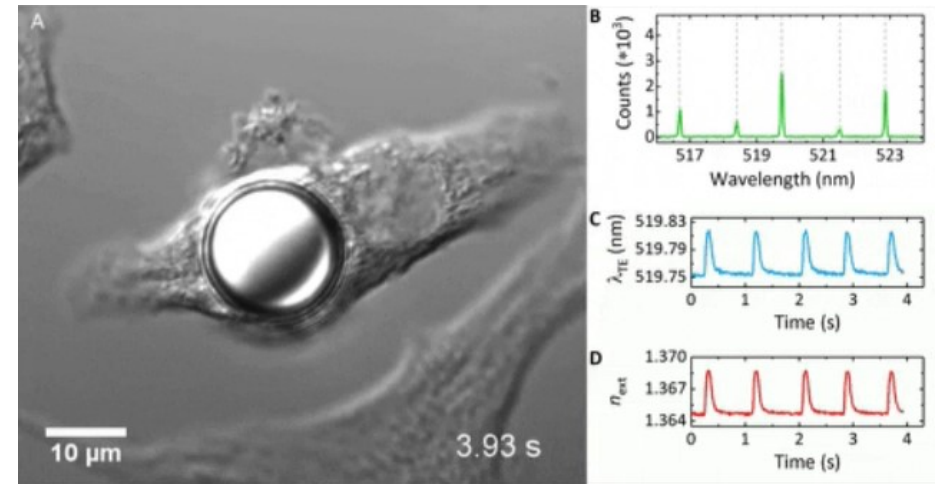

The wavelength of light emitted from a laser inside a heart cell (left) shifts by a few hundredths of a percent (right middle graph) each time the heart cell contracts. Schubert and his colleagues think that this shift comes from a change in the refractive index of the heart cell (right bottom graph). The lasers used by the team emit five different wavelengths of light (right top graph), each of which the team tracked.

Credit: M. Schubert/University of St Andrews

when the circling light hits an excited dye molecule, as it gains additional photons from stimulated emission-just as in a normal laser. Some of this circulating light leaks out, producing a laser signal that can be 1000 times brighter than the signal from conventional fluorescence-microscopy techniques, making it much easier to spot. It is this leaked light that the team studied in their experiments.

The team placed a bead next to an isolated mouse heart cell, which "swallowed" up the bead. When they exposed the cell to light, they observed that the wavelengths of the laser light shifted up and down each time the cell contracted and expanded. At around $0.03 \%$ of each wavelength, these shifts were tiny, but they were clearly visible on the spectrometer, Schubert says.

He and his colleagues explained these shifts as arising from local refractive-index changes in proteins called myofibrils. These proteins, which look like long, skinny rods, contract each time a heart cell beats. This contraction causes the local protein density in the cell to temporarily increase, upping the cell's refractive index. When the proteins relax and lengthen again, the opposite happens. Using a confocal microscope, Schubert and his colleagues tracked length changes of the myofibrils closest to the bead and found that their shortening and lengthening correlated perfectly with the wavelength change.

The team also performed experiments with live zebrafish hearts and with slices taken from rat heart tissue. For the latter, they showed that they could detect the beating signals from beads through $400 \mu \mathrm{m}$ of tissue. "Other people have tried to monitor refractive-index changes deep inside tissues without success," Schubert said. "Here we have shown that we can really do that with our laser beads."

Schubert and his colleagues are also working on smaller lasers made from a semiconductor called indium gallium phosphide. Their initial experiments indicate that these nanometer-sized disk lasers can-like the bead lasers-be used to track changes in a cell's refractive index. Because of their small size, tens of these lasers could be inserted into a single cell, allowing for localized measurements within the cell, Schubert says.

Now that they know the technique works, the team is planning to do experiments in lab-grown 3D heart tissues. For example, the researchers are considering placing their lasers inside embryos to monitor the biomechanical properties of a heart as it develops.

Katherine Wright is the Deputy Editor of Physics. 